\title{
Search for Novel Target Molecules for the Effective Treatment or Prevention of Colorectal Cancer
}

\author{
Masahiko Tsujii \\ Department of Gastroenterology and Hepatology, Osaka University Graduate School of Medicine, Suita, Japan
}

\section{Key Words}

CRC prevention - Chemoprevention, target molecules .

Polypectomy $\cdot$ Micronutrients

\begin{abstract}
The 5-year survival rate is more than $70 \%$ for patients without distant metastasis, while it is $10-20 \%$ for those with distant metastatic disease, suggesting the essential development of effective perioperative therapy. In a combination of cetuximab and FOLFIRI, the rate of conversion to resectable liver metastases is about $30 \%$, and oncogenic activation of intracellular signaling pathway downstream of epidermal growth factor receptor, like KRAS mutations, is an important mechanism of resistance to anti-epidermal growth factor receptor therapy. Other molecules in oncogenic signal transduction and microRNA are attracting attention as candidates for new molecular targets. A cancer stem cell theory has been advocated even in gastrointestinal tract cancer and thought to be involved in multidrug resistance via ATP-binding cassette transport and active DNA repair. Despite newlydevised therapeutic ways, curative treatment of advanced colorectal cancer (CRC) leading to development of prevention is still difficult. Currently, the most effective preventive strategy is endoscopic polypectomy. A most recent report showed removal of adenoma was conducive to a $77 \%$ lower risk for CRC after 10 years. However, another means of prevention needs to be developed for familial adenomatous
\end{abstract}

\section{KARGER}

Fax +41613061234 E-Mail karger@karger.ch www.karger.com
(C) 2012 S. Karger AG, Basel

0012-2823/12/0852-0099\$38.00/0

Accessible online at:

www.karger.com/dig polyposis and hereditary non-polyposis CRC. Adding to the micronutrients, several key molecules have been investigated as target candidates for chemoprevention. A deeper understanding of the biology of CRC has been expanded, which is resulting in the development of novel therapies.

Copyright $\odot 2012$ S. Karger AG, Basel

\section{New Potential Treatment for Advanced Colorectal Cancer}

Colorectal cancer (CRC) is the third most common cancer in the Western world. Nearly $25 \%$ of patients with CRC present with synchronous liver metastases at the time of initial diagnosis. Recurrences after resection of the primary tumor occur in the liver in $60-70 \%$ of cases. Surgery of CRC with metastases remains the best treatment modality, but only $20-35 \%$ of patients turn out to be suitable candidates for surgery; even after surgical resection, the long-term survival rate is unacceptably low [1]. The 5-year survival rate is, at best, $10-20 \%$ for patients with distant metastatic disease. On the other hand, the 5 -year survival rate for patients without lymph node metastasis is more than $80 \%$, and is $70 \%$ even for those with lymph node metastasis if they do not have distant metastases. Therefore, the development of effective perioperative therapy is essential. Currently, molecular target therapy, including cetuximab and bevacizumab, is used for 


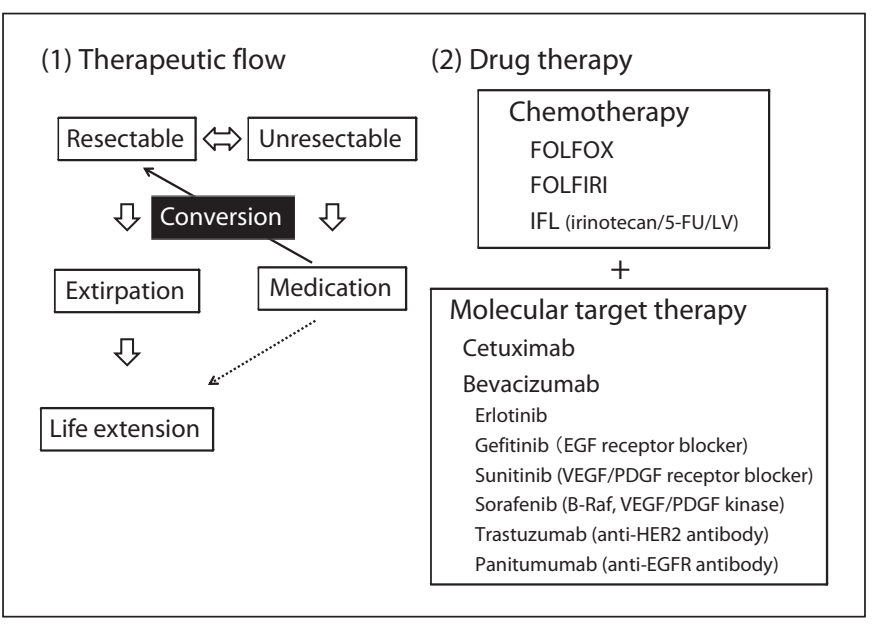

Fig. 1. Present therapy for advanced CRC: (1) Therapeutic flow: recently, even unresectable CRC and (2) Medication for advanced CRC: systemic chemotherapy with or without biological therapy resulted in increased response, resection, and survival rates in patients with unresectable CRC.

the treatment of CRC in addition to FOLFOX or FOLFIRI chemotherapy (fig. 1). Above all, cetuximab shows a great antitumor effect. The overall response rate of a combination of cetuximab and FOLFIRI in the treatment of patients with unresectable CRC liver metastases is about $40 \%$, and the rate of conversion to resectable liver metastases is about $30 \%$, showing a survival advantage [2]. Cetuximab and panitumumab are anti-epidermal growth factor receptor (anti-EGFR) monoclonal antibodies; these antibodies are successful in treating patients with an activated EGFR mutant. Oncogenic activation of intracellular signaling pathways downstream of EGFR is an important mechanism of resistance to anti-EGFR antibodies. KRAS mutations are found in approximately $40 \%$ of the cases and demonstrate a major predictive marker of resistance to these antibodies. Recently, Raf-MEK kinases and mammalian target of rapamycin (mTOR) are attracting attention as candidates for new molecular targets.

MicroRNA (miRNA) is a non-coding RNA; one miRNA regulates the expression of more than 100 genes. miRNA plays an important role in drug resistance, and hence clinical trials are designed with miRNA as a new target. Godlewski et al. [3] reported that Bmi-1 (an oncogene playing a role in the epigenetic silencing of certain genes) expression was significantly upregulated and miR128 expression was downregulated in a panel of patient glioblastoma specimens as compared to in the normal brain, and miR-128 expression significantly inhibited glioblastoma cell proliferation in vitro and glioblastoma xenograft growth in vivo. It has been shown that miRNAs also affect cell sensitivity to different chemotherapeutic agents, and although in vivo validation results are awaited, miRNAs have shown very strong chemosensitization effects. Holleman et al. [4] identified miR-135a as a miRNA related to paclitaxel resistance and showed that in paclitaxel-resistant cell lines, miR-135a inhibition sensitized resistant cell lines to paclitaxel-induced cell death.

Multidrug resistance genes such as MDR1 (ABCB1), $M R P 1$ ( $A B C C 1$ ), and $A B C G 2$ have been identified. Recently, however, a cancer stem cell theory has been advocated even in solid tumor-like gastrointestinal tract cancer. Cancer stem cells are marked by characteristics such as self-renewal, pluripotency, tumor-initiating capacity, ATP-binding cassette (ABC) transport, and active DNA repair; the latter two characteristics are thought to be involved in multidrug resistance. Dallas et al. [5] prepared 5-fluorouracil (5-FU)-resistant or oxaliplatin-resistant CRC cells (HT29) by exposing cells to increasing doses of each drug in order to achieve resistance at clinically relevant doses. Both resistant cells were cross-resistant to 5-FU or oxaliplatin and expressed the stem cell marker CD133/CD44. These results indicate that multidrug-resistant cells contain many cancer stem cells. We also prepared 5-FU- or oxaliplatin-resistant HT29 cells. These cells did not show significant cross-resistance but showed strong expression of stem cell markers. In addition, the expression of epithelial mesenchymal transition-related genes was upregulated, whereas that of cytoskeleton-associated genes was dysregulated; further, the motility potential of these resistant cells was upregulated.

These results led us to develop novel molecular targets directed at key molecules critical for cancer stem cell biology.

\section{Promising Ideas for CRC Prevention}

Recently, new therapeutic ways with miRNA or epigenetically active reagents have been devised, but at present it is still difficult to cure advanced CRC, which is bringing home the importance of methods of prevention against CRC.

Approximately $25 \%$ of CRC cases occur in individuals with a family history of the disease; moreover, $5 \%$ of these cases are caused by the genetic syndromes familial adenomatous polyposis or hereditary non-polyposis CRC. The risk of CRC is also higher in individuals with inflammatory bowel disease, but the latter is implicated in only 
$5-15 \%$ of CRC cases. The remaining $75 \%$ of CRC cases are sporadic; this suggests that it is very difficult to identify individuals at a high risk of CRC.

\section{Polypectomy}

At present, endoscopic polypectomy is the most effective preventive strategy for CRC. The most recent report analyzing a total of 1,688 case patients with CRC and 1,932 participants aged 50 years or older revealed that colonoscopy with detection and removal of adenomas in the preceding 10 years was associated with a $77 \%$ lower risk for CRC; moreover, the adjusted odds ratio for right-sided CRC and left-sided CRC was 0.44 and 0.16 , respectively. However, CRC development in the case of familial adenomatous polyposis (APC) and hereditary non-polyposis CRC cannot be prevented by polypectomy, and hence, another means of prevention needs to be developed.

\section{Chemoprevention}

Chemoprevention is the use of natural or laboratorymade substances to prevent a disease such as cancer. With regard to CRC, several substrates have been investigated as candidates for chemopreventive reagents (table 1).

\section{Micronutrients}

Some micronutrients have been included as candidates for a long time. The effectiveness of calcium in the prevention of a colorectal tumor has also been investigated for a long time. The most recent meta-analysis shows that supplemental calcium (1,200-2,000 mg/day) effectively prevents adenoma recurrence in a population with a history of adenomas but did not affect the relative risk of advanced adenoma and CRC [6]. $\beta$-Carotene supplementation has not been shown to have any beneficial effect on cancer prevention [7]. With regard to vitamin D, scientific evidence supports its role in the decrease of CRC incidence, and possibly in mortality [8]. With regard to folic acid, its high level intake reduces the risk of CRC; further, there is no evidence to suggest that dietary fortification or supplementation with folic acid increases the risk of CRC [9]. Recently, other micronutrients have gained particular interest. Among these, zinc is reported to have the pharmaceutical potential for chemoprevention via p53 induction, apoptosis signal-regulated protein kinase-dependent cell damage, mitochondria dysfunction, and apoptosis/necrosis/autophagy induction.

Target Molecules for Chemoprevention

Some molecules have been shown to play an important role in cancer prevention:

Search for Novel Target Molecules for the Effective Treatment or Prevention of CRC
Table 1. High-potential chemopreventive agents

Micronutrients
Calcium
$\beta$-Carotene
Vitamin D
Folic acid
Zinc
Molecular target for chemoprevention
Nrf2
Metformin
Notch inhibitors (Aes)
Cyclooxygenase-2 inhibitors
GST- $\pi$ inhibitor, GST-activated cytotoxic prodrugs
Anti-obesity, diet, exercise
PPAR- $\gamma$

Nrf2/ARE. Nrf2/ARE is a carcinogen detoxification enzyme, which has antioxidant and anti-inflammatory functions. Nrf-2 plays a central role in protection against oxidative damage. Under unstressed conditions, Nrf2 is constantly ubiquitinated by the Clu3-Keap 1 ubiquitin E3 ligase complex and rapidly degraded. Upon exposure to oxidative stress, reactive cysteine residues of Keapl become modified, which leads to a decline in E3 ligase activity, stabilization of Nrf2, and induction of cytoprotective genes. Glycogen synthase kinase- $3 \beta$ also regulates Nrf2 expression [10].

Metformin. Recent epidemiological evidence has revealed that metformin users are at a lower risk of cancer than users of insulin or insulin secretagogue. In a recent clinical study, short-term use of metformin reduced aberrant crypt foci formation in non-diabetic patients [11], and in vivo and in vitro studies showed that metformin inhibited cell proliferation. The major molecular targets of metformin are 5'-AMP-activated protein kinase (AMPK) signaling and mTOR pathways, which are central to the regulation of cellular energy homeostasis and play a crucial role in the control of energy homeostasis and cell proliferation. Metformin improves glycemic control and endothelial function, decreases inflammatory activity, and regulates immune function. Metformin seems to be a practical option as an antitumor and chemopreventive agent [12].

Notch Signal Inhibitors. Aes (amino-terminal enhancer of split) was identified as one of the genes responsible for a high metastatic potential. Aes expression is reduced in liver metastases as compared to in primary colon tumor in mice and humans. It inhibits Notch signaling that stimulates transendothelial migration. Notch signaling inhibition

Digestion 2012;85:99-102 
suppresses malignant alterations such as tumor invasion and intravasation in Aes and Apc double-knockout mice. Therefore, Notch signaling inhibitors have shown promise as preventive agents of colon cancer metastasis [13].

Cyclooxygenase-2 (COX-2) Inhibitors. COX-2 is overexpressed in CRC tissues and plays a crucial role in antiapoptosis, angiogenesis, invasion, and metastatic potential. The Food and Drug Administration (FDA) has approved the use of celecoxib for the chemoprevention of $\mathrm{CRC}$ in patients with familial adenomatous polyposis. It is anticipated that COX-2 inhibitors are associated with an elevated risk of cardiovascular diseases.

Glutathione-S-Transferase (GST)-Pai Inhibitor and GST-Activated Cytotoxic Prodrugs [14]. GSTs are overexpressed in cancer tissues and are recognized as negative modulators of apoptosis.

Anti-Obesity. Obesity increases the risk of CRC because of the consequently elevated leptin level [15]. A high fat diet is involved in the promotion of CRC via hyperinsulinemia-induced activation of the c-Jun $\mathrm{N}$-terminal kinase (JNK) pathway [15]. Regular exercise can prevent the onset of CRC via decreased tumor necrosis factor- $\alpha$ and inducible nitric oxide synthase expression [12].
Peroxisomal Proliferator-Activated Receptor (PPAR)- $\gamma$. In vitro and in vivo studies show the possibility of PPAR$\gamma$ agonists to suppress tumor growth, although it is reported that PPAR- $\gamma$ agonists promoted colon cancer under certain conditions [16].

\section{Conclusion}

Key signaling pathways that play key roles in the initiation and progression of CRC have been elucidated in succession. Significant advances have been made in understanding the biology of CRC. We review such data on cancer biology and provide important insights for the development of novel therapies that target specific growth factor receptors and/or certain critical signal transduction pathways. These targeted therapies can then be used alone or in combination with standard cytotoxic chemotherapy regimens.

\section{Disclosure Statement}

The author declares that no financial or other conflicts of interest exist in relation to the content of the article.

\section{References}

1 Wieser M, Sauerland S, Arnold D, Schmiegel W, Reinacher-Schick A: Peri-operative chemotherapy for the treatment of resectable liver metastases from colorectal cancer: a systematic review and meta-analysis of randomized trials. BMC Cancer 2010;10:309.

-2 Min BS, Kim NK, Ahn JB, Roh JK, Kim KS, Choi JS, Cha SH, Kim H: Cetuximab in combination with 5 -fluorouracil, leucovorin and irinotecan as a neoadjuvant chemotherapy in patients with initially unresectable colorectal liver metastases. Onkologie 2007;30:637-643.

- 3 Godlewski J, Nowicki MO, Bronisz A, Williams S, Otsuki A, Nuovo G, Raychaudhury A, Newton HB, Chiocca EA, Lawler S: Targeting of the Bmi-1 oncogene/stem cell renewal factor by microRNA-128 inhibits glioma proliferation and self-renewal. Cancer Res 2008;68:9125-9130.

4 Holleman A, Chung I, Olsen RR, Kwak B, Mizokami A, Saijo N, Parissenti A, Duan Z, Voest EE, Zetter BR: miR-135a contributes to paclitaxel resistance in tumor cells both in vitro and in vivo. Oncogene 2011;30:4386-4398.

-5 Dallas NA, Xia L, Fan F, Gray MJ, Gaur P, van Buren G 2nd, Samuel S, Kim MP, Lim SJ, Ellis LM: Chemoresistant colorectal cancer cells, the cancer stem cell phenotype, and increased sensitivity to insulin-like growth factor-I receptor inhibition. Cancer Res 2009;69:1951-1957.
6 Carroll C, Cooper K, Papaioannou D, Hind D, Pilgrim H, Tappenden P: Supplemental calcium in the chemoprevention of colorectal cancer: a systematic review and metaanalysis. Clin Ther 2010;32:789-803.

7 Druesne-Pecollo N, Latino-Martel P, Norat T, Barrandon E, Bertrais S, Galan P, Hercberg S: Beta-carotene supplementation and cancer risk: a systematic review and metaanalysis of randomized controlled trials. Int J Cancer 2010;127:172-184.

8 Davis CD, Milner JA: Vitamin D and colon cancer. Expert Rev Gastroenterol Hepatol 2011;5:67-81.

-9 Stevens VL, McCullough ML, Sun J, Jacobs EJ, Campbell PT, Gapstur SM: High levels of folate from supplements and fortification are not associated with increased risk of colorectal cancer. Gastroenterology 2011;141:98-105.

10 Taguchi K, Motohashi H, Yamamoto M: Molecular mechanisms of the Keap1-Nrf2 pathway in stress response and cancer evolution. Genes Cells 2011;16:123-140.

-11 Hosono K, Endo H, Takahashi H, Sugiyama M, Sakai E, Uchiyama T, Suzuki K, Iida H, Sakamoto Y, Yoneda K, Koide T, Tokoro C, Abe Y, Inamori M, Nakagama H, Nakajima A: Metformin suppresses colorectal aberrant crypt foci in a short-term clinical trial. Cancer Prev Res (Phila) 2010;3:1077-1083.
12 Aoi W, Naito Y, Takagi T, Kokura S, Mizushima K, Takanami Y, Kawai Y, Tanimura Y, Hung LP, Koyama R, Ichikawa H, Yoshikawa $\mathrm{T}$ : Regular exercise reduces colon tumorigenesis associated with suppression of iNOS. Biochem Biophys Res Commun 2010;399:14-19.

-13 Sonoshita M, Aoki M, Fuwa H, Aoki K, Hosogi H, Sakai Y, Hashida H, Takabayashi A, Sasaki M, Robine S, Itoh K, Yoshioka K, Kakizaki F, Kitamura T, Oshima M, Taketo MM: Suppression of colon cancer metastasis by Aes through inhibition of Notch signaling. Cancer Cell 2001;19:125-137.

$\checkmark 14$ Miyanishi K, Takayama T, Ohi M, Hayashi T, Nobuoka A, Nakajima T, Takimoto R, Kogawa K, Kato J, Sakamaki S, Niitsu Y: Glutathione S-transferase-pi overexpression is closely associated with K-ras mutation during human colon carcinogenesis. Gastroenterology 2001;121:865-874.

15 Endo H, Hosono K, Uchiyama T, Sakai E, Sugiyama M, Takahashi H, Nakajima N, Wada K, Takeda K, Nakagama H, Nakajima A: Leptin acts as a growth factor for colorectal tumours at stages subsequent to tumour initiation in murine colon carcinogenesis. Gut 2011;60:1363-1371.

16 Thompson EA: PPAR $\gamma$ physiology and pathology in gastrointestinal epithelial cells. Mol Cells 2007;24:167-176. 